TRANSACTIONS OF THE

AMERICAN MATHEMATICAL SOCIETY

Volume 356, Number 8, Pages 3143-3166

S 0002-9947(03)03337-3

Article electronically published on November 4, 2003

\title{
CORE VERSUS GRADED CORE, AND GLOBAL SECTIONS OF LINE BUNDLES
}

\author{
EERO HYRY AND KAREN E. SMITH
}

\begin{abstract}
We find formulas for the graded core of certain $\mathfrak{m}$-primary ideals in a graded ring. In particular, if $S$ is the section ring of an ample line bundle on a Cohen-Macaulay complex projective variety, we show that under a suitable hypothesis, the core and graded core of the ideal of $S$ generated by all elements of degrees at least $N$ (for some, equivalently every, large $N$ ) are equal if and only if the line bundle admits a non-zero global section. We also prove a formula for the graded core of the powers of the unique homogeneous maximal ideal in a standard graded Cohen-Macaulay ring of arbitrary characteristic. Several open problems are posed whose solutions would lead to progress on a non-vanishing conjecture of Kawamata.
\end{abstract}

\section{INTRODUCTION}

When does an ample line bundle on a smooth projective variety have a nonzero global section? In this paper, we show that this question is equivalent to a fundamental problem in commutative algebra regarding the equality of core and graded core for a certain associated homogeneous ideal. This builds on the project begun in [16], where it was shown that a sufficiently good algebraic understanding of the graded core can be used to show the existence of global sections of line bundles. This paper essentially treats the converse: we show that the existence of non-zero sections gives rise to nice formulas for cores.

The core of an ideal in a Noetherian commutative ring is the intersection of all its reductions - that is, the intersection of all subideals having the same integral closure. The core first arose in the work of Rees and Sally 25] because of its connection with Briançon-Skoda theorems, and has recently been the subject of active investigation in commutative algebra; see [11, 2, 3, 16, 24, 13.

For a homogeneous ideal in a graded ring, it is also natural to consider its graded core, namely, the intersection of all its homogeneous reductions. The core and graded core of a homogeneous ideal are both homogeneous ideals, and there is an obvious inclusion of the core in the graded core. A natural question is: when does equality hold? This question arose in the work [16] in finding sections of line bundles, and has also been considered by Huneke and Trung in [13] for purely algebraic reasons.

Received by the editors January 30, 2003.

2000 Mathematics Subject Classification. Primary 13A30; Secondary 13A15, 14 B15.

The first author's research was supported by the National Academy of Finland, project number 48556 .

The second author's research was partially supported by the Clay Foundation and by the US National Science Foundation Grant DMS 00-70722.

(C)2003 American Mathematical Society 
Quite generally, the core and graded core are equal for homogeneous ideals generated by elements of the same degree (see Lemma 3.3 for a precise statement). On the other hand, there are examples of ideals having so few homogeneous reductions that it is easy to see the core is strictly smaller. But what can be said for ideals having many graded reductions?

In this paper we answer this question for a specific type of ideal in a section ring, while providing one answer to the opening question about sections of line bundles. Specifically, we prove a formula for the graded core (see Theorem 3.1 and Corollary 3.10) which, when combined with the formula for the core from [16, yields the following result.

Theorem 1.1. Let $\mathcal{L}$ be an ample invertible sheaf on an irreducible projective variety $X$ over a field of characteristic zero, and let

$$
S=\bigoplus_{n \geq 0} H^{0}\left(X, \mathcal{L}^{n}\right)
$$

be the corresponding section ring. Assume that $S$ is Gorenstein. Fix $N \gg 0$, and let $I=S_{\geq N}$ be the ideal generated by all elements of degree at least $N$. Then gradedcore $(I)=\operatorname{core}(I)$ if and only if $\mathcal{L}$ has a non-trivial global section. Furthermore, in this case,

$$
\text { gradedcore }(I)=\operatorname{core}(I)=S_{\geq N d+a+1},
$$

where $d$ is the dimension of $S$ and $a$ is its a-invariant 1

This theorem makes it easy to construct examples of homogeneous ideals with abundant homogeneous reductions where core and graded core differ. On the other hand, one might also hope to use the theorem to algebraically prove the existence of global sections of particular line bundles - indeed, this is our motivation for studying the core.

Theorem 1.1follows from a more general result which is valid without the Gorenstein assumption on $S$. To motivate this, recall that in [16] it was shown that from the point of view of projective geometry, one should really look at cores inside the canonical module of $S$. That is, the object in which we are interested is the module gradedcore $\left(J \omega_{S}\right)$, defined as the intersection in $\omega_{S}$ of the submodules $J \omega_{S}$ as $J$ ranges over all homogeneous reductions of $I$. With this definition, we prove the following, which specializes immediately to Theorem 1.1 if $S$ happens to be Gorenstein.

Theorem 1.2. Let $\mathcal{L}$ denote an ample invertible sheaf on an irreducible projective variety $X$ of positive dimension $d-1$ over a field of characteristic zero, and let

$$
S=\bigoplus_{n \geq 0} H^{0}\left(X, \mathcal{L}^{n}\right)
$$

be the corresponding section ring. Assume that the local cohomology modules $H_{\mathfrak{m}}^{i}(S)$ supported at the unique homogeneous maximal ideal of $S$ vanish in negative degrees for $i<d$. Fix $N \gg 0$, and let $I=S_{\geq N}$ be the ideal generated by all elements of degree at least $N$. Then $H^{0}(X, \mathcal{L}) \neq 0$ if and only if gradedcore $\left(I \omega_{S}\right)=\operatorname{core}\left(I \omega_{S}\right)$. Furthermore, in this case, we have

$$
\text { gradedcore }\left(I \omega_{S}\right)=\operatorname{core}\left(I \omega_{S}\right)=\left[\omega_{S}\right]_{\geq d n+1} .
$$

\footnotetext{
${ }^{1}$ By definition, the $a$-invariant of $S$ is defined by $a=-\min \left\{n \mid\left[\omega_{S}\right]_{n} \neq 0\right\}$.
} 
Our methods also yield the following formula for the core in standard graded rings of arbitrary characteristic.

Theorem 1.3. Let $(S, \mathfrak{m})$ be a standard graded reduced Cohen-Macaulay ring of dimension $d$ over an infinite field of arbitrary characteristic, and let a denote its a-invariant. Then for all $N \geq 1$,

$$
\operatorname{core}\left(\mathfrak{m}^{N}\right)=\operatorname{gradedcore}\left(\mathfrak{m}^{N}\right)=\mathfrak{m}^{N d+a+1}
$$

Similar formulas appear in [16, §6], [24] and [13. Note that Theorem [1.3] does not require the ground field to have characteristic zero, as in these other formulas. Indeed, according to [24 Example 5.9], in general, the core of the maximal ideal can depend on the characteristic.

Our original motivation for studying the core was the following remarkable conjecture of Kawamatd2: an ample line bundle $\mathcal{L}$ on a smooth variety $X$ always has a non-zero global section provided that $\mathcal{L} \otimes \omega_{X}^{-1}$ is also ample. In [16], we showed that this conjecture follows from a conjectured formula for the graded core generalizing a formula due to Huneke and Swanson. As a corollary to the main theorem of the current paper, we see that in fact this conjectured formula for the graded core is equivalent to Kawamata's conjecture; see Conjecture 3.12

The contents of the paper are as follows. Section 2 contains a list of properties about section rings. The main work is done in Section 3, where the main result, Theorem [3.1, is proven. Section 4 contains the proof of Theorem 1.3. Section 5 contains a list of commutative algebra questions and detailed remarks motivating them. Progress towards any of these questions may lead to progress towards Kawamata's conjecture or a number of other related conjectures in birational algebraic geometry. We believe that commutative algebraists ought to be studying section rings more, and we hope our questions help stimulate research in this direction. We also hope that the geometric connections will help other researchers to appreciate the core.

\section{SECTION RINGS}

Let $X$ be a projective scheme over a field and let $\mathcal{L}$ be an ample invertible sheaf on $X$. The section ring of the pair $(X, \mathcal{L})$ is the $\mathbb{N}$-graded ring

$$
S=\bigoplus_{n \in \mathbb{N}} H^{0}\left(X, \mathcal{L}^{n}\right)
$$

This is a finitely generated graded ring, and there is a natural isomorphism $\operatorname{Proj} S \cong$ $X$ under which the sheaves on $\operatorname{Proj} S$ given by the graded module $S(n)$ correspond to the invertible sheaves $\mathcal{L}^{n}$. (Here $S(n)$ denotes the graded cyclic $S$-module generated in degree $-n$.) The ring $S$ is reduced if and only if $X$ is reduced, and is a domain if and only if $X$ is reduced and irreducible. In this case, furthermore, $S$ is normal if and only if $X$ is normal. Moreover, the sheaf given by the graded canonical module $\omega_{S}$ of $S$ corresponds to $\omega_{X}$.

Section rings are much nicer than arbitrary graded rings. They share many of the properties of standard graded rings (that is, those generated in degree one) and should be thought of as a natural generalization of this standardly considered

\footnotetext{
${ }^{2}$ This was also posed as a question by Ambro in [1].
} 
case. A normal section ring is standard graded if and only if the line bundle $\mathcal{L}$ is very ample and projectively normal; thus the passage from standard graded to section rings in commutative algebra is analogous to the passage from very ample to ample line bundles in algebraic geometry. Under this analogy, the powers of the homogeneous maximal ideal of a standard graded ring correspond to the ideals generated by elements of degrees at least $N$ in a section ring. It is these ideals whose cores are crucial in understanding sections of $\mathcal{L}$.

For future reference, we summarize here a few of the nice properties of section rings. All of these properties can fail for an arbitrary graded ring.

Proposition 2.1 (Properties of Section Rings). Let $S$ be the section ring of a projective variety, and let $\mathfrak{m}$ denote its unique homogeneous maximal ideal. Then:

(1) The section ring $S$ has depth at least two (or depth one in the case of a section ring of dimension one); that is, $H_{\mathfrak{m}}^{0}(S)=0$ and, if $\operatorname{dim} S>1$, $H_{\mathfrak{m}}^{1}(S)=0$ as well.

(2) The sheaf corresponding to the graded module $S(1)$ is an invertible sheaf on Proj $S$.

(3) The sheaves corresponding to the graded modules $S(n)$ are invertible on Proj $S$ for all $n$.

(4) There exist homogeneous non-zero-divisors $x_{1}, \ldots, x_{r}$ generating an $\mathfrak{m}$ primary ideal such that the $\mathbb{Z}$-graded ring $S_{x_{i}}$ is isomorphic to the ring $\left[S_{x_{i}}\right]_{0}\left[t, t^{-1}\right]$, where $t$ is an indeterminate of degree one and $\left[S_{x_{i}}\right]_{0}$ is the subring of the localization ring $S_{x_{i}}$ consisting of degree zero elements.

(5) For any homogeneous element $x$ and any $n \in \mathbb{Z}$, the $n$-th graded piece of $S_{x}$ is a rank one projective module over the subring $\left[S_{x}\right]_{0}$.

(6) For all $n \gg 0$, the elements of degree $n$ generate an $\mathfrak{m}$-primary ideal.

(7) For all $n \gg 0, S_{n}^{p}=S_{n p}$ for all $p \geq 1$.

(8) For all $p, q \gg 0, S_{p} S_{q}=S_{p+q}$.

(9) The ring $S$ has isolated singularity (respectively, isolated non-rationally singular point, isolated non-Cohen-Macaulay point, isolated non-Gorenstein point, et cetera) if and only if the variety Proj $S$ is non-singular (respectively, rationally singular, Cohen-Macaulay, Gorenstein, et cetera.)

(10) The Hilbert function $n \mapsto \operatorname{dim} S_{n}$ is polynomial for $n \gg 0$.

(11) If $\operatorname{Proj} S$ is smooth (respectively, has rational singularities, is Cohen-Macaulay, et cetera), then also Proj $S^{\natural}$ is smooth (respectively, has rational singularities, is Cohen-Macaulay, et cetera), where $S^{\natural}=\bigoplus_{n \geq 0} S_{\geq n}$ is the Rees ring of the natural filtration $\left\{S_{\geq n}\right\}_{n \in \mathbb{N}}$.

Furthermore, Properties (2), (3), and (4) each characterize normal section rings among all finitely generated normal graded domains.

Proof. We only sketch the proofs, since all can be found in the literature. The original source for section rings is [9, Section 4.5]. In [5], normal graded rings are studied quite generally and shown to be rings of sections for ample $\mathbb{Q}$-Cartier Weil divisors - the section ring case corresponds to the divisor actually being Cartier.

Property 1 follows by computing $H_{\mathfrak{m}}^{i}(S)$ from the extended Čech complex

$$
0 \longrightarrow S \longrightarrow \bigoplus S_{x_{i}} \longrightarrow \bigoplus_{i<j} S_{x_{i} x_{j}} \longrightarrow \ldots
$$


where $x_{0}, \ldots, x_{d}$ is a homogeneous system of parameters for $S$. This leads to an exact sequence

$$
0 \longrightarrow H_{\mathfrak{m}}^{0}(S) \longrightarrow S \longrightarrow \bigoplus_{n \in \mathbb{Z}} H^{0}\left(X, \mathcal{L}^{n}\right) \longrightarrow H_{\mathfrak{m}}^{1}(S) \longrightarrow 0 .
$$

By definition of $S$, then, we see that both $H_{\mathfrak{m}}^{0}(S)$ and $H_{\mathfrak{m}}^{1}(S)$ must vanish. (The computation when $S$ has dimension one is similar.)

Properties (2) and (3) are immediate, since the sheaf associated to $S(n)$ is isomorphic to $\mathcal{L}^{n}$, which is invertible. These properties characterize section rings by the main theorem of [5].

Property (4) is essentially a restatement of (3). The $x_{i}$ are chosen so that the open sets $U_{i}=\operatorname{Spec}\left[S_{x_{i}}\right]_{0}$ in $\operatorname{Proj} S$ where $x_{i}$ does not vanish are a trivializing cover for $\mathcal{L}$. Then $\mathcal{L}\left(U_{i}\right)=\left[S_{x_{i}}\right]_{n}$ is a free $\mathcal{O}_{\operatorname{Proj} S}\left(U_{i}\right)=\left[S_{x_{i}}\right]_{0}$-module of rank one. Letting $t$ be a generator for $\mathcal{L}\left(U_{i}\right)$, it follows that $t^{n}$ is a generator for $\mathcal{L}^{n}$. The isomorphism follows. Conversely, if the isomorphism holds, then each $\mathcal{L}^{n}\left(U_{i}\right)$ is clearly free of rank one. Property (5) is essentially Property (4), stated for any arbitrary cover of Proj $S$ rather than only for a cover that trivializes $\mathcal{L}$. See also [29].

Properties (6), (7) and (8) follow from the definition of ampleness. In particular, Property (6) follows because $\mathcal{L}^{n}$ is globally generated for all large $n$ : then $\mathcal{L}^{n}$ has a set of global sections that do not simultaneously vanish, which means that the ideal of $S$ they generate is $\mathfrak{m}$-primary. Property (7) follows since $\mathcal{L}^{n}$ is very ample and projectively normal for all large $n$. Property (8) follows from the Künneth formula; see, for example, [21, Example 1.2.18].

Property (9) follows immediately from Property (4), because the map $A \longrightarrow$ $A\left[t, t^{-1}\right]$ is smooth for any ring $A$.

Property (10) follows from Serre vanishing. Indeed, the function $n \mapsto \chi\left(X, \mathcal{L}^{n}\right)$ is a polynomial in $n$ (its precise form being given by the Riemann-Roch formula), where $\chi\left(X, \mathcal{L}^{n}\right)=h^{0}\left(X, \mathcal{L}^{n}\right)-h^{1}\left(X, \mathcal{L}^{n}\right)+\cdots \pm h^{d}\left(X, \mathcal{L}^{n}\right)$ [28], 20]. For $n \gg 0$, Serre vanishing ensures that all the higher cohomology terms are zero.

Finally, Property (11) is essentially treated, for example, in [16, Paragraph 6.2.1] or [9, Section 8.7.3]. The point is that Proj $S^{\natural}$ is isomorphic to the total space of the line bundle $\mathcal{L}$ over $X$. Thus there is a smooth map $\operatorname{Proj} S^{\natural} \longrightarrow \operatorname{Proj} S$ making all the nice properties of $\operatorname{Proj} S$ pass to $\operatorname{Proj} S^{\natural}$.

We will use most of these properties without explicit mention in the next section. In fact, the only properties we really use are (6), (7) and (8), but because section rings are a natural and important class, we prefer to state our results for them.

\section{THE MAIN THEOREM}

For a ring $S$, recall that core $\left(I \omega_{S}\right)$ denotes the intersection of all submodules $J \omega_{S}$ of $\omega_{S}$, where $J$ is a reduction of $I$. Similarly, when $I$ is a homogeneous ideal in a graded ring $S$, gradedcore $\left(I \omega_{S}\right)$ is the corresponding intersection over all homogeneous reductions $J$ of $I$. Our goal is to prove the following theorem.

Theorem 3.1. Let $S$ be an equidimensional section ring of dimension $d \geq 2$ and characteristic zero, and assume that $\operatorname{Proj} S$ is Cohen-Macaulay. Fix $N \gg 0$, and 
let $I=S_{\geq N}$ be the ideal generated by all elements of degree at least $\left.N\right\}$ Then,

$$
\text { gradedcore }\left(I \omega_{S}\right)=\bigoplus_{i \in \mathbb{Z} ; S_{i}=0}\left[\omega_{S}\right]_{N d-i}
$$

as a graded submodule of $\omega_{S}$.

Note that in particular (taking negative values of $i$ ), gradedcore $\left(I \omega_{S}\right)$ contains the submodule $\left[\omega_{S}\right]_{\geq N d+1}$ generated by elements of degrees at least $N d+1$. In the situation of [16, Corollary 6.4.1], this submodule is precisely core $\left(I \omega_{S}\right)$. But if $S$ is "missing" a component of degree $i$, then the graded core picks up elements of degree $N d-i$. In particular, Theorem 1.2 from the introduction is an immediate corollary of Theorem 3.1 .

We now turn to the proof of Theorem 3.1 We begin with a lemma that reduces this problem to a core computation for a slightly different ideal.

Lemma 3.2. Let $S$ be an equidimensional section ring such that $\operatorname{Proj} S$ is CohenMacaulay. Fix $N \gg 0$, and let $I$ denote the ideal generated by elements of degree at least $N$. Then gradedcore $\left(I \omega_{S}\right)=\operatorname{core}\left(I^{\prime} \omega_{S}\right)$, where $I^{\prime}$ denotes the ideal of $S$ generated by all elements of degree precisely $N$.

Proof. Because $N \gg 0$, we know that $S$ has systems of parameters of degree $N$. In particular, $I^{\prime}$ is a reduction of $I$, and we must have

$$
\text { gradedcore }\left(I \omega_{S}\right) \subset \text { gradedcore }\left(I^{\prime} \omega_{S}\right) \text {. }
$$

On the other hand, every homogeneous minimal reduction $J$ of $I$ is generated by elements of degree $N$ (see [16, Proposition 2.1.3]). So

$$
\operatorname{gradedcore}\left(I \omega_{S}\right)=\operatorname{graded} c o r e\left(I^{\prime} \omega_{S}\right) \text {. }
$$

But as $I^{\prime}$ is generated by homogeneous elements of the same degree $N$, Lemma 3.3 below ensures that core $\left(I^{\prime} \omega_{S}\right)=\mathfrak{a}_{1} \omega_{S} \cap \ldots \cap \mathfrak{a}_{r} \omega_{S}$, where $\mathfrak{a}_{1}, \ldots, \mathfrak{a}_{r}$ are homogeneous minimal reductions of $I$. To apply Lemma 3.3, note that since both modules are $\mathfrak{m}$-primary, we can check equality after localization. The hypothesis that $S_{\mathfrak{m}}$ is a generalized Cohen-Macaulay ring is satisfied because $S$ is equidimensional with (at worst) an isolated non-Cohen-Macaulay point. The proof that gradedcore $\left(I^{\prime} \omega_{S}\right)=$ core $\left(I^{\prime} \omega_{S}\right)$ is complete.

Lemma 3.3. Let $(A, \mathfrak{m})$ be a generalized Cohen-Macaulay local ring of dimension $d$ containing its residue field $k=A / \mathfrak{m}$. Let $I \subset A$ be an $\mathfrak{m}$-primary ideal. Let $\left\{a_{1}, \ldots, a_{\mu}\right\}$ be a set of generators for $I$. If $I \subset \mathfrak{m}^{N}$, where $N \gg 0$, then there exist minimal reductions $\mathfrak{a}_{1}, \ldots, \mathfrak{a}_{t}$ of $I$ such that $\operatorname{core}\left(I \omega_{A}\right)=\mathfrak{a}_{1} \omega_{A} \cap \ldots \cap \mathfrak{a}_{t} \omega_{A}$ and for each $\mathfrak{a}_{h}$ there is a generating set $\left\{b_{h 1}, \ldots, b_{h d}\right\}$, where

$$
b_{h i}=\sum_{j=0}^{\mu} \lambda_{h i j} a_{j} \quad \text { with } \quad \lambda_{h i j} \in k \quad \text { for } \quad h=1, \ldots, t ; i=1, \ldots, d .
$$

A similar statement holds also for cores of ideals rather than cores inside the canonical module.

\footnotetext{
${ }^{3}$ The precise value required for $N$ is not very important; what is used is that $\mathcal{L}^{n}$ is very ample, and that the higher cohomology modules of $\omega_{X} \otimes \mathcal{L}^{n}$ vanish for all $n \geq N$.
} 
Proof. This is a slight generalization of a special case of [2, Theorem 4.5]. We must remove the hypothesis there that $A$ is Cohen-Macaulay and make some minor adjustments to replace the core ideal by the core submodule in $\omega_{A}$.

Because there exists an $r$ such that every reduction of $I$ contains $I^{r+1}$, it follows that $\omega_{A} / \operatorname{core}\left(I \omega_{S}\right)$ has finite length. So there are in any case finitely many reductions $J_{1}, \ldots, J_{t}$ of $I$ such that core $\left(I \omega_{A}\right)=J_{1} \omega_{A} \cap \ldots \cap J_{t} \omega_{A}$. The CohenMacaulayness of $A$ was used in the proof of [2, Theorem 4.5] only in the proof of [2] Lemma 4.3], which established the equality $l_{A}\left(I \omega_{A} / \mathfrak{a}_{i} \omega_{A}\right)=l_{A}\left(I \omega_{A} / J_{i} \omega_{A}\right)$. We will instead establish this equality under the assumption that $A$ is a generalized Cohen-Macaulay ring.

Let $M$ be a generalized Cohen-Macaulay $A$-module, meaning that the local cohomology modules $H_{m}^{i}(M)$ are all of finite length, for $i<\operatorname{dim} M$. Recall (see, for example, 31]) that an $\mathfrak{m}$-primary ideal $\mathfrak{b}$ of $A$ is called standard with respect to $M$ if every system of parameters $\left(b_{1}, \ldots, b_{d}\right)$ for $A / \operatorname{Ann} M$ contained in $\mathfrak{b}$ satisfies $l_{A}\left(M /\left(b_{1}, \ldots, b_{d}\right) M\right)-e\left(b_{1}, \ldots, b_{d} ; M\right)=\mathbb{I}(M)$, where $\mathbb{I}(M)$ is the Buchsbaum invariant $\sum_{i=0}^{\operatorname{dim} M-1}\left({ }_{i}^{\operatorname{dim} M-1}\right) l_{A}\left(H_{\mathfrak{m}}^{i}(M)\right)$ of $M$. By [10, Satz 3.3], there always exists an $N \gg 0$ such that every ideal $\mathfrak{b}$ contained in $\mathfrak{m}^{N}$ is standard with respect to $M$. Our assumption that $N$ is large, together with [8, Theorem 3.17], implies that $I$ is standard with respect to $\omega_{A}$. So

$$
\begin{aligned}
l_{A}\left(I \omega_{A} / J_{i} \omega_{A}\right) & =l_{A}\left(\omega_{A} / J_{i} \omega_{A}\right)-l_{A}\left(\omega_{A} / I \omega_{A}\right) \\
& =e\left(J_{i} ; \omega_{A}\right)+\mathbb{I}\left(\omega_{A}\right)-l_{A}\left(\omega_{A} / I \omega_{A}\right) \\
& =e\left(\mathfrak{a}_{i} ; \omega_{A}\right)+\mathbb{I}\left(\omega_{A}\right)-l_{A}\left(\omega_{A} / I \omega_{A}\right)=l_{A}\left(I \omega_{A} / \mathfrak{a}_{i} \omega_{A}\right),
\end{aligned}
$$

as needed.

To compute core $\left(I^{\prime} \omega_{S}\right)$, we will apply the main technical theorem of [16]. First, we need to recall some notation from Section 3.1 of that paper.

Notation 1 (Adjoint Type Modules). Let $(A, \mathfrak{m})$ be a local ring which is a homomorphic image of a Gorenstein local ring, and let $I$ be any ideal of $A$ of positive height. Set $Y=\operatorname{Proj} A[I t]$, where $A[I t]$ is the Rees algebra of $I$. For each integer $p$, we define the $p$-th adjoint type module by

$$
\Omega_{p}=\Gamma\left(Y, I^{p} \omega_{Y}\right) .
$$

Note that the adjoint type modules depend on the ideal $I$, although this dependence is suppressed from the notation.

Clearly, $\Omega_{p+1} \subset \Omega_{p}$ and $I \Omega_{p} \subset \Omega_{p+1}$ for all $p \in \mathbb{Z}$. Recall that $\Omega_{0}$ can be considered as a submodule of $\omega_{A}$ by means of the trace homomorphism $\Omega_{0} \longrightarrow \omega_{A}$ for the blowing up map $Y \rightarrow \operatorname{Spec} A$. (When $I$ has height at least two, the trace homomorphism can be defined in an elementary way as follows: Let $U \subset Y$ be the non-empty open set of $Y$ isomorphic under the blowing up to Spec $A \backslash \mathbb{V}(I)$. Then

$$
\Omega_{0}=\Gamma\left(Y, \omega_{Y}\right) \stackrel{\text { restr. }}{\longrightarrow} \Gamma\left(U, \omega_{Y}\right) \cong \Gamma\left(\operatorname{Spec} A \backslash \mathbb{V}(I), \omega_{A}\right)=\omega_{A},
$$

with the first arrow being the restriction map and the last equality holding because $\omega_{A}$ satisfies Serre's $S_{2}$ condition.) In particular, we get an $I$-filtration $\omega_{A} \supset \Omega_{1} \supset$ $\Omega_{2} \ldots$. As explained in [16, Paragraph 2.6.2], for positive values of $p$, there is a natural identification of $\Omega_{p}$ with the $p$-th graded component of the graded canonical module $\omega_{A[I t]}$ of $A[I t]$. 
The proof of Theorem 3.1 is based on the following result:

Theorem 3.4. Let $S$ be a finitely generated $\mathbb{N}$-graded domain of dimension $d \geq 2$ containing the rational numbers, and let $\mathfrak{m}$ denote its homogeneous maximal ideal. Let $I$ be an $\mathfrak{m}$-primary ideal generated by elements of all the same degree. Assume that for any homogeneous reduction $J$ of $I$,

$$
J \omega_{S} \cap \Omega_{d-1}=J \Omega_{d-2}
$$

as submodules of $\omega_{S}$, where $\Omega_{p}$ is the $p$-th adjoint type module of $I$ as defined in (11). Then gradedcore $\left(I \omega_{S}\right) \subset \Omega_{d}$.

Proof. This is a graded version of the main technical theorem of [16], and is a direct consequence of Lemma 3.3.1 and Theorem 3.4.1 there.

Thus, combining Lemma 3.2 and Theorem 3.4 we see that to compute the graded core of $I \omega_{S}$, we need to verify (2) for the adjoint type modules associated to $I^{\prime}$, where $I^{\prime}$ is the ideal of $S$ generated by the elements of degree $N$. We use the notation

$$
\Omega_{p}^{\prime}=\Gamma\left(Y^{\prime}, I^{\prime p} \Omega_{Y^{\prime}}\right)
$$

where $Y^{\prime}=\operatorname{Proj} S\left[I^{\prime} t\right]$ for these modules.

Notation $2\left(S^{\natural}\right.$ and $\left.S^{\dagger}\right)$. Recall the "natural" construction from [9, Section 8.7.3]. Given a graded ring $S$, we form the graded ring

$$
S^{\natural}=\bigoplus_{p \geq 0} S_{\geq p}
$$

where $S_{\geq p}$ is the ideal generated by all elements of $S$ of degrees at least $p$. The ring $S^{\natural}$ is the Rees ring corresponding to the "natural" filtration $\left\{S_{\geq p}\right\}_{p \geq 0}$, and the corresponding associated graded ring recovers $S$. Note that if $I=S_{\geq N}$, where $N \gg 0$ is chosen in such a way that $S_{\geq p N}=\left(S_{\geq N}\right)^{p}$ for all $p \geq 1$, then the Veronese subring $\left(S^{\natural}\right)^{(N)}$ coincides with the Rees algebra $S[I t]$.

We define a graded subring $S^{\dagger}$ of $S^{\natural}$ by setting

$$
S^{\dagger}=\bigoplus_{p \geq 0} S_{p} S
$$

where $S_{p} S$ denotes the ideal of $S$ generated by elements of degree precisely $p$. If $N \gg 0$ is chosen as above, then the Veronese subring $\left(S^{\dagger}\right)^{(N)}$ coincides with the Rees ring $S\left[I^{\prime} t\right]$, where $I^{\prime}$ is the ideal of $S$ generated by the elements of degree exactly $N$.

Note that $S^{\natural}$ is finite and birational over $S^{\dagger}$ (and if $S$ is normal, then $S^{\natural}$ is the normalization of $S^{\dagger}$ ). Both rings have dimension $d+1$, where $d$ is the dimension of $S$. Furthermore, both rings have natural bigradings:

$$
S^{\dagger}=\bigoplus_{p, q \geq 0} S_{p} S_{q} \quad \text { and } \quad S^{\natural}=\bigoplus_{p, q \geq 0} S_{p+q} .
$$

We will make much use of these bigradings. There is also a third grading, namely, the "total degree," whose $n$ graded components are defined as follows:

$$
\left[S^{\dagger}\right]_{n}=\bigoplus_{p, q \geq 0, p+q=n} S_{p} S_{q} \quad \text { and } \quad\left[S^{\natural}\right]_{n}=\bigoplus_{p, q \geq 0, p+q=n} S_{p+q} .
$$


We compute the $p$-th adjoint type module $\Omega_{p}^{\prime}$ of $I^{\prime}$ by interpreting it as the appropriate graded piece of the graded canonical module of $S^{\dagger}$. With this in mind, the point is to prove the following theorem.

Theorem 3.5. Let $S$ be an equidimensional section ring of dimension $d \geq 2$, with Proj $S$ Cohen-Macaulay. Let $G$ denote the set of (positive and negative) integers $n$ for which $S_{n}$ is zero. Then, for any positive $p$ such that $\left[H_{\mathfrak{m}}^{d-1}(S)\right]_{n}=0$ for all $n<-p$, we have

$$
\left[\omega_{S^{\dagger}}\right]_{p}=\bigoplus_{-q \in G}\left[\omega_{S}\right]_{p+q}
$$

Furthermore, if $S$ has negative a-invariant, then we also get the following description for non-positive $p$ :

$$
\left[\omega_{S^{\dagger}}\right]_{p}= \begin{cases}{\left[\omega_{S}\right]_{\geq p+1}} & \text { if }-p \in G \\ 0 & \text { otherwise }\end{cases}
$$

Remark 3.1. Note that the set $G$ of Theorem 3.5 contains all the negative integers. In particular, $\left[\omega_{S^{\dagger}}\right]_{p}$ contains the submodule $\left[\omega_{S}\right]_{\geq p+1}$. Since $G$ contains only finitely many positive integers, $\left[\omega_{S^{\dagger}}\right]_{p}$ differs from $\left[\omega_{S}\right]_{\geq p+1}$ only by a vector space of finite dimension.

Remark 3.2. If $S$ is Cohen-Macaulay or if Proj $S$ has rational singularities (and is over a field of characteristic zero), then $\left[H_{\mathfrak{m}}^{d-1}(S)\right]$ vanishes in all negative degrees, so the cohomological hypothesis of Theorem 3.5 is satisfied automatically. (When Proj $S$ has rational singularities, this follows from the Kodaira vanishing theorem; see [12].) Furthermore, since Proj $S$ is Cohen-Macaulay, it is satisfied for all sufficiently large $p$.

Proof. We will study the graded pieces of $\omega_{S^{\dagger}}$ by comparison with those of $\omega_{S \natural}$, which are easier to understand.

First note that the finite birational map $S^{\dagger} \hookrightarrow S^{\natural}$ induces a natural inclusion (the trace map) $\omega_{S^{\natural}} \hookrightarrow \omega_{S^{\dagger}}$. We wish to understand the cokernel. We do this by studying the kernel of the dual map.

Let $\mathfrak{M}$ and $\mathfrak{N}$ denote the homogeneous maximal ideals of $S^{\natural}$ and $S^{\dagger}$ respectively. Note that the radical of $\mathfrak{N} S^{\natural}$ is $\mathfrak{M}$. Since $\operatorname{dim} S^{\natural} / S^{\dagger}<d+1$, we obtain a surjective homomorphism

$$
\psi: H_{\mathfrak{N}}^{d+1}\left(S^{\dagger}\right) \longrightarrow H_{\mathfrak{N}}^{d+1}\left(S^{\natural}\right)=H_{\mathfrak{M}}^{d+1}\left(S^{\natural}\right) .
$$

This surjection is dual to the trace map $\omega_{S^{\natural}} \hookrightarrow \omega_{S^{\dagger}}$. Thus we need to understand the kernel of $\psi$.

To this purpose, set

$$
\mathfrak{N}^{\prime}=\bigoplus_{p \geq 1, q \geq 0} S_{p, q}^{\dagger}, \quad \mathfrak{N}^{\prime \prime}=\bigoplus_{p \geq 0, q \geq 1} S_{p, q}^{\dagger} \quad \text { and } \quad \mathfrak{N}^{+}=\bigoplus_{p \geq 1, q \geq 1} S_{p, q}^{\dagger}
$$

Observe that $\mathfrak{N}^{\prime}+\mathfrak{N}^{\prime \prime}=\mathfrak{N}$ and $\mathfrak{N}^{\prime} \cap \mathfrak{N}^{\prime \prime}=\mathfrak{N}^{+}$. The Mayer-Vietoris sequences for the cohomology of $S^{\dagger}$ and $S^{\natural}$ respectively yield the commutative diagram

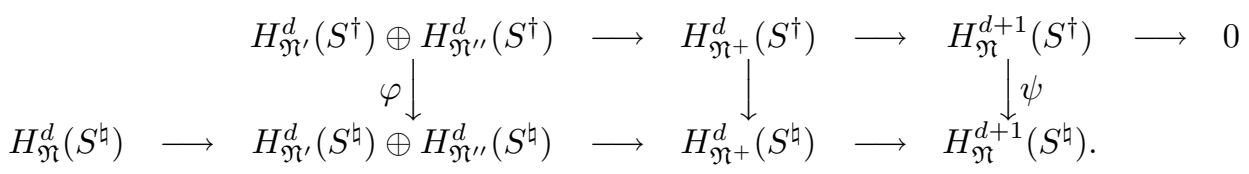


Now, the module $H_{\mathfrak{N}}^{d}\left(S^{\natural}\right)$ vanishes in negative $p$-degrees (and hence in negative $q$-degrees by symmetry). Indeed, the Sancho de Salas sequence (see [23] or [16] Paragraph 2.6]) yields an exact sequence of graded modules

$$
\cdots \bigoplus_{p \in \mathbb{Z}} H_{E}^{d-1}\left(\mathcal{O}_{Y}(p)\right) \longrightarrow H_{\mathfrak{N}}^{d}\left(S^{\natural}\right) \longrightarrow \bigoplus_{p \in \mathbb{N}} H_{m}^{d}\left(S_{\geq p}\right) \longrightarrow \ldots,
$$

where $Y=\operatorname{Proj} S^{\natural}$ and $E$ is the closed fiber of the blowing up map $Y \longrightarrow \operatorname{Spec} S$. For negative $p$, then, the vanishing of $\left[H_{\mathfrak{N}}^{d}\left(S^{\natural}\right)\right]_{p}$ follows from the vanishing of $H_{E}^{d-1}\left(\mathcal{O}_{Y}(p)\right)$. By duality, this is equivalent to the vanishing of $H^{1}\left(Y, \omega_{Y}(-p)\right)$. This vanishing in turn is a consequence of Lemma 3.6 below.

Also, the middle vertical arrow in the commutative diagram above is an isomorphism. Indeed, because $S_{p+q}=S_{p} S_{q}$ for all $p, q \gg 0$, we have $\left(\mathfrak{N}^{+}\right)^{n}\left(S^{\natural} / S^{\dagger}\right)=0$ for $n \gg 0$, whence $H_{\mathfrak{N}^{+}}^{i}\left(S^{\natural} / S^{\dagger}\right)=0$ for all $i>0$. A look at the long exact cohomology sequence corresponding to the exact sequence

$$
0 \longrightarrow S^{\dagger} \longrightarrow S^{\natural} \longrightarrow S^{\natural} / S^{\dagger} \longrightarrow 0
$$

then shows that $H_{\mathfrak{N}^{+}}^{i}\left(S^{\dagger}\right)=H_{\mathfrak{N}^{+}}^{i}\left(S^{\natural}\right)$ for all $i>1$.

We are now in a position to use the snake lemma to compute the cokernel of $\psi$ in degree $p<0$. For $p<0$, we get an isomorphism

$$
[\operatorname{Ker} \psi]_{p, q} \cong[\operatorname{Coker} \varphi]_{p, q} \text {. }
$$

Thus we need to understand the map $\varphi$ in degree $p<0$.

To understand $\varphi$, we first look at the local cohomology modules of $S^{\dagger}$ and $S^{\natural}$ with supports in $\mathfrak{N}^{\prime}$ and $\mathfrak{N}^{\prime \prime}$. Note that the homogeneous maximal ideal $\mathfrak{m}$ of $S$ extends to $\mathfrak{N}^{\prime}$ under the homomorphism $S \longrightarrow S^{\dagger}$ obtained by considering $S$ as the subring of the bigraded ring $S^{\dagger}$ concentrated in degrees $(p, 0)$, for $p \geq 0$. Thus

$$
H_{\mathfrak{N}^{\prime}}^{d}\left(S^{\natural}\right)=H_{\mathfrak{m}}^{d}\left(S^{\natural}\right)=\bigoplus_{q \geq 0} H_{\mathfrak{m}}^{d}\left(S_{\bullet, q}^{\natural}\right)=\bigoplus_{q \geq 0} H_{\mathfrak{m}}^{d}\left(S_{\geq q}\right),
$$

where $S_{\bullet, q}^{\natural}$ denotes the graded $S$-module $\bigoplus_{p \geq 0} S_{p+q}=S_{\geq q}$. Now, using the long exact sequence of cohomology corresponding to the exact sequence

$$
0 \longrightarrow S_{\bullet, q}^{\natural} \longrightarrow S(q) \longrightarrow \bigoplus_{p=-q}^{-1} S_{p+q} \longrightarrow 0,
$$

we get

$$
H_{\mathfrak{N}^{\prime}}^{d}\left(S^{\natural}\right)=\bigoplus_{q \geq 0} H_{\mathfrak{m}}^{d}(S(q))
$$

and so by symmetry also

$$
H_{\mathfrak{N}^{\prime \prime}}^{d}\left(S^{\natural}\right)=\bigoplus_{p \geq 0} H_{\mathfrak{m}}^{d}(S(p)) .
$$

It is now clear that $H_{\mathfrak{N}^{\prime}}^{d}\left(S^{\natural}\right)$ vanishes in negative $q$-degrees and that $H_{\mathfrak{N}^{\prime \prime}}^{d}\left(S^{\natural}\right)$ vanishes in negative $p$-degrees.

So the cokernel of $\varphi$ in degrees $p<0$ is isomorphic to the cokernel of the natural map

$$
H_{\mathfrak{N}^{\prime}}^{d}\left(S^{\dagger}\right) \longrightarrow H_{\mathfrak{N}^{\prime}}^{d}\left(S^{\natural}\right)
$$


induced by the inclusion of $S^{\dagger}$ in $S^{\natural}$. Since $H_{\mathfrak{N}^{\prime}}^{d+1}\left(S^{\natural}\right)=H_{\mathfrak{m}}^{d+1}\left(S^{\natural}\right)$ is zero, this cokernel is precisely

$$
H_{\mathfrak{N}^{\prime}}^{d}\left(S^{\natural} / S^{\dagger}\right) \cong \bigoplus_{q \geq 0} H_{\mathfrak{m}}^{d}\left(S_{\geq q} / S_{q} S\right)
$$

Hence, in degree $(p, q)$, we have

$$
[\text { Coker } \varphi]_{p, q}=\left[H_{\mathfrak{m}}^{d}\left(S_{\geq q} / S_{q} S\right)\right]_{p} .
$$

Now if $S_{q} \neq 0$, then $S_{\geq q} / S_{q} S$ is an $S$-module of dimension strictly less than $d$, and so $\left[H_{\mathfrak{m}}^{d}\left(S_{\geq q} / S_{q} S\right)\right]_{p}=0$. On the other hand, if $S_{q}=0$, then

$$
\left[H_{\mathfrak{m}}^{d}\left(S_{\geq q} / S_{q} S\right)\right]_{p}=\left[H_{\mathfrak{m}}^{d}\left(S_{\geq q}\right)\right]_{p} \cong\left[H_{\mathfrak{m}}^{d}(S(q))\right]_{p}=\left[H_{\mathfrak{m}}^{d}(S)\right]_{p+q} .
$$

Summarizing, we have for negative $p$

$$
[\operatorname{Coker} \varphi]_{p, q}= \begin{cases}{\left[H_{\mathfrak{m}}^{d}(S)\right]_{p+q}} & \text { if } q \in G ; \\ 0 & \text { otherwise. }\end{cases}
$$

It thus follows that for positive $p$ there is an exact sequence

$$
0 \longrightarrow\left[\omega_{S^{\natural}}\right]_{p} \longrightarrow\left[\omega_{S^{\dagger}}\right]_{p} \longrightarrow Q_{p} \longrightarrow 0
$$

where

$$
Q_{p, q}= \begin{cases}{\left[\omega_{S}\right]_{p+q}} & \text { if }-q \in G \\ 0 & \text { otherwise }\end{cases}
$$

Finally, Lemma 3.7 below ensures that the $p$-th graded piece of $\omega_{S^{\natural}}$ is $\left[\omega_{S}\right]_{\geq p+1}$, so this formula for the cokernel $Q_{p}$ immediately implies the desired formula for the positively graded pieces of $\omega_{S^{\dagger}}$. The proof of Theorem 3.5 in the case of positive values of $p$ is complete.

To prove Theorem 3.5 for non-positive $p$ under the additional hypothesis that $S$ has negative $a$-invariant, we use Lemma 3.8 below. This guarantees that the module $H_{\mathfrak{N}}^{d}\left(S^{\natural}\right)$ vanishes also for non-negative $p$. We can then use the snake lemma as above to compute the cokernel of $\varphi$ also for $p \geq 0$. Doing so yields the desired conclusion in a similar way.

Lemma 3.6. Let $S$ be an equidimensional section ring of dimension $d \geq 2$ such that Proj $S$ is Cohen-Macaulay. Assume that $H_{\mathfrak{m}}^{d-1}(S)$ vanishes in degrees less than $-p$. Let $Y$ denote Proj $S^{\natural}$ with its natural polarization. Then $H^{1}\left(Y, \omega_{Y}(p)\right)=0$.

Proof. First note that in the important special case where Proj $S$ has rational singularities and is defined over a field of characteristic zero, the result follows for all $p \geq 0$ immediately from the Grauert-Riemenschneider vanishing theorem. Indeed, in this case $Y=\operatorname{Proj} S^{\natural}$ has rational singularities. Let $Z \stackrel{\nu}{\rightarrow} Y$ be a resolution. By the Grauert-Riemenschneider vanishing theorem, the direct image sheaves

$$
R^{j} \nu_{*}\left(\omega_{Z} \otimes \nu^{*} \mathcal{O}_{Y}(p)\right)=R^{j} \nu_{*} \omega_{Z} \otimes \mathcal{O}_{Y}(p)
$$

vanish for $j \geq 1$. Thus the corresponding spectral sequence degenerates, and we have an isomorphism

$$
H^{i}\left(Z, \omega_{Z} \otimes \nu^{*} \mathcal{O}_{Y}(p)\right) \cong H^{i}\left(Y, \nu_{*} \omega_{Z} \otimes \mathcal{O}_{Y}(p)\right)
$$

for all $i \geq 0$. Now, because $Y$ has rational singularities, $\nu_{*} \omega_{Z}=\omega_{Y}$; and so the required vanishing follows from a standard vanishing theorem applied to the resolution $Z \longrightarrow \operatorname{Spec} S$ (see [4]). 
We now assume that Proj $S$ is Cohen-Macaulay and $H_{\mathfrak{m}}^{d-1}(S)$ vanishes in degrees less than $-p$. We interpret $Y$ as the total space bundle over $X$ for the line bundle $\mathcal{L}^{-1}=\mathcal{O}_{X}(-1)$ defining the section ring $S$. The map $f: Y \longrightarrow X$ is affine and smooth, with $\omega_{Y}=f^{*} \omega_{X} \otimes f^{*} \mathcal{O}_{X}(1)$. Computing as in the proof of [16. Proposition 6.2.1], we see that

$$
\begin{aligned}
H^{1}\left(Y, \omega_{Y}(p)\right) & =H^{1}\left(Y, f^{*}\left(\omega_{X}(p+1)\right)\right)=H^{1}\left(X, f_{*} \mathcal{O}_{Y} \otimes \omega_{X}(p+1)\right) \\
& =\bigoplus_{n \geq p+1} H^{1}\left(X, \omega_{X}(n)\right) .
\end{aligned}
$$

By duality, this module vanishes by our assumption that $H_{\mathfrak{m}}^{d-1}(S)$ vanishes in degrees less than $-p$.

Lemma 3.7. If $S$ is a section ring of an equidimensional projective variety, then for positive $p$,

$$
\left[\omega_{S^{\natural}}\right]_{p}=\left[\omega_{S}\right]_{\geq p+1} .
$$

Proof. It has been proven in [16, Proposition 6.2.1] that if $Y=\operatorname{Proj} S^{\natural}$, then $\Gamma\left(Y, \omega_{Y}(p)\right)=\left[\omega_{S}\right]_{\geq p+1}$ for all $p \in \mathbb{Z}$. We now use the general fact that we can identify $\left[\omega_{S^{\natural}}\right]_{p}$ with $\Gamma\left(Y, \omega_{Y}(p)\right)$ for all $p \geq 1$ (see, for example, [16, Paragraph 2.6.2]).

Lemma 3.8. With notation as above, suppose that the section ring $S$ has negative a-invariant. Then $H_{\mathfrak{N}}^{d}\left(S^{\natural}\right)$ vanishes in non-negative $p$ (and hence $q$ ) degrees.

Proof. Recall that for any filtration $\left\{I_{i}\right\}_{i \in \mathbb{N}}$ of a ring $S$ we have two fundamental short exact sequences,

$$
0 \longrightarrow R^{+} \longrightarrow R \longrightarrow S \longrightarrow 0 \text { and } 0 \longrightarrow R^{+}(1) \longrightarrow R \longrightarrow G \longrightarrow 0,
$$

where $R$ is the Rees ring $\bigoplus_{i \in \mathbb{N}} I_{i}, R^{+}$is its irrelevant ideal $\bigoplus_{i>0} I_{i}$, and $G$ is the associated graded ring $\bigoplus I_{i} / I_{i+1}$. In particular, applying this to the natural filtration of $S$, we get short exact sequences

$$
0 \longrightarrow\left(S^{\natural}\right)^{+} \longrightarrow S^{\natural} \longrightarrow S \longrightarrow 0 \quad \text { and } \quad 0 \longrightarrow\left(S^{\natural}\right)^{+}(1) \longrightarrow S^{\natural} \longrightarrow S \longrightarrow 0,
$$

where in the first sequence the module $S$ is viewed as concentrated in degree zero, while in the second sequence it has its usual grading.

The long exact sequence arising from the first sequence yields an isomorphism $H_{\mathfrak{N}}^{d}\left(\left(S^{\natural}\right)^{+}\right) \cong H_{\mathfrak{N}}^{d}\left(S^{\natural}\right)$ in all non-zero $p$ degrees. The second sequence gives an exact sequence

$$
H_{\mathfrak{N}}^{d}\left(\left(S^{\natural}\right)^{+}(1)\right) \longrightarrow H_{\mathfrak{N}}^{d}\left(S^{\natural}\right) \longrightarrow H_{\mathfrak{m}}^{d}(S) .
$$

Therefore, if $p \geq 0$, our hypothesis gives a surjection

$$
\left[H_{\mathfrak{N}}^{d}\left(\left(S^{\natural}\right)^{+}(1)\right)\right]_{p} \longrightarrow\left[H_{\mathfrak{N}}^{d}\left(S^{\natural}\right)\right]_{p} .
$$

Since $\left[H_{\mathfrak{N}}^{d}\left(\left(S^{\natural}\right)^{+}(1)\right)\right]_{p} \cong\left[H_{\mathfrak{N}}^{d}\left(\left(S^{\natural}\right)^{+}\right)\right]_{p+1} \cong\left[H_{\mathfrak{N}}^{d}\left(S^{\natural}\right)\right]_{p+1}$ for all non-negative $p$, we then get surjections

$$
\left[H_{\mathfrak{N}}^{d}\left(S^{\natural}\right)\right]_{p+1} \longrightarrow\left[H_{\mathfrak{N}}^{d}\left(S^{\natural}\right)\right]_{p}
$$

for all $p \geq 0$. This completes the proof, since evidently $H_{\mathfrak{N}}^{d}\left(S^{\natural}\right)$ vanishes in large degrees. 
We now have all the ingredients we need to prove Theorem 3.1 .

Proof of Theorem 3.1. For each integer $p$, set $\Omega_{p}^{\prime}=\Gamma\left(Y^{\prime}, I^{\prime p} \omega_{Y^{\prime}}\right)$, where $I^{\prime}$ is the ideal of $S$ generated by all elements of degree $N$ and $Y^{\prime}=\operatorname{Proj} S\left[I^{\prime} t\right]$. We first claim that for $p \geq 1$,

$$
\Omega_{p}^{\prime}=\bigoplus_{i \in \mathbb{Z} ; S_{i}=0}\left[\omega_{S}\right]_{p N-i} .
$$

Note here that $i$ ranges through all integers such that $S_{i}$ is zero, including all negative integers. The grading is not given by $i$, but by the inherited grading from $\omega_{S}$.

To see why the claim is true, for each $p \geq 1$, we can identify $\Omega_{p}^{\prime}$ with the $p$-th homogeneous component of the graded canonical module of $S\left[I^{\prime} t\right]$. Since $S\left[I^{\prime} t\right]=$ $\left(S^{\dagger}\right)^{(N)}$, we also have $\omega_{S\left[I^{\prime} t\right]}=\left(\omega_{S^{\dagger}}\right)^{(N)}$. Now by Theorem 3.5, we have

$$
\Omega_{p}^{\prime}=\left[\omega_{S\left[I^{\prime} t\right]}\right]_{p}=\left[\omega_{S^{\dagger}}\right]_{N p}=\bigoplus_{i \in \mathbb{N} ; S_{i}=0}\left[\omega_{S}\right]_{N p-i}
$$

(The required vanishing needed for Theorem 3.5 holds by taking $N$ to be very large.)

We first show the inclusion gradedcore $\left(I \omega_{S}\right) \subset \Omega_{d}^{\prime}$. By Lemma 3.2 ,

$$
\operatorname{graded} \operatorname{core}\left(I \omega_{S}\right)=\operatorname{core}\left(I^{\prime} \omega_{S}\right) \text {. }
$$

To compute the latter, we appeal to Theorem 3.4 which requires that the inclusion

$$
\Omega_{d-1}^{\prime} \cap J \omega_{S}=J \Omega_{d-2}^{\prime}
$$

holds for every homogeneous minimal reduction of $I$. To verify (4), take any homogeneous element $w$ in $\Omega_{d-1}^{\prime} \cap J \omega_{S}$, say of degree $n$. Since $J$ is necessarily generated by elements of degree $N$, we can write $w=s_{1} w_{1}+\ldots+s_{r} w_{r}$ where $s_{1}, \ldots, s_{r}$ are homogeneous elements of degree $N$ in $J$ and $w_{1}, \ldots, w_{r} \in\left[\omega_{S}\right]_{n-N}$. We want to show that the $w_{i}$ are in $\Omega_{d-2}^{\prime}$. For this we use (3). If $n \geq(d-1) N+1$, then $\left[\Omega_{d-2}^{\prime}\right]_{n-N}=\left[\omega_{S}\right]_{n-N}$, and we are done. On the other hand, when $n<(d-1) N+1$, there is nothing to prove unless $n=(d-1) N-i$ for some $i$ such that $S_{i}=0$. But in this case $\left[\Omega_{d-2}^{\prime}\right]_{n-N}=\left[\omega_{S}\right]_{n-N}$, too. Thus gradedcore $\left(I \omega_{S}\right) \subset \Omega_{d}^{\prime}$, as needed.

The reverse inclusion is an immediate application of Lemma 3.9] below (since there are only finitely many positive $i$ such that $S_{i}=0$ ). Note that this lemma implies that when $S_{i}=0$ or when $i$ is negative, we have $\left[\omega_{S}\right]_{N d-i} \subset J \omega_{S}$ for every homogeneous reduction $J$ of $I$.

Lemma 3.9. Let $\mathcal{L}$ be an ample invertible sheaf on a projective variety $X$ of dimension $d-1$. Fix any $N$ so that $\mathcal{L}^{N}$ is globally generated and so that

$$
H^{j}\left(X, \mathcal{L}^{\otimes N(d-1-j)-i} \otimes \omega_{X}\right)=0
$$

for $j$ in the range $0<j<d-1$, and

$$
H^{j}\left(X, \mathcal{L}^{\otimes N(d-j)-i} \otimes \omega_{X}\right)=0
$$

for positive $j$. If $X$ satisfies Serre's $S_{2}$ condition, then $H^{0}\left(X, \mathcal{L}^{\otimes i}\right)=0$ if and only if for any set $x_{1}, \ldots, x_{d}$ of d global generators for $\mathcal{L}^{N}$, the natural inclusion

$$
\sum_{j=1}^{d} x_{j} H^{0}\left(X, \omega_{X} \otimes \mathcal{L}^{\otimes[N(d-1)-i]}\right) \subset H^{0}\left(X, \omega_{X} \otimes \mathcal{L}^{\otimes(N d-i)}\right)
$$


is an equality. Similarly, for any $N \gg i$,

$$
\sum_{j=1}^{d} x_{j} H^{0}\left(X, \mathcal{L}^{\otimes[N(d-1)+i]}\right) \subset H^{0}\left(X, \mathcal{L}^{\otimes(N d+i)}\right)
$$

is an equality if and only if $H^{d-1}\left(X, \mathcal{L}^{\otimes i}\right)=0$.

Proof. The first statement is essentially [16, Lemma 6.1.2]. The second is proved similarly:

Consider the Koszul complex determined by the $x_{i}$ on $X$, where $\mathcal{O}_{X}(N)$ denotes $\mathcal{L}^{N}$ :

$$
0 \longrightarrow \mathcal{O}_{X}(-N d) \longrightarrow \ldots \longrightarrow \mathcal{O}_{X}(-N)^{\oplus d} \stackrel{\left(x_{1}, \ldots, x_{d}\right)}{\longrightarrow} \mathcal{O}_{X} \longrightarrow 0 .
$$

Since the $x_{i}$ do not all simultaneously vanish on $X$, this complex is exact. Now tensor with the invertible sheaf $\mathcal{O}_{X}(N d+i)$ to get an exact sequence

$$
0 \longrightarrow \mathcal{O}_{X}(i) \longrightarrow \ldots \longrightarrow \mathcal{O}_{X}(N(d-1)+i)^{\oplus d} \stackrel{\left(x_{1}, \ldots, x_{d}\right)}{\longrightarrow} \mathcal{O}_{X}(N d+i) \longrightarrow 0 .
$$

Using the same argument as in [16, Lemma 6.1.2], we see that the map

$$
\Gamma\left(X, \mathcal{O}_{X}(N(d-1)+i)^{\oplus d}\right) \stackrel{\left(x_{1}, \ldots, x_{d}\right)}{\longrightarrow} \Gamma\left(X, \mathcal{O}_{X}(N d+i)\right)
$$

is surjective if and only if

$$
H^{d-1}\left(X, \mathcal{O}_{X}(i)\right) \text { vanishes. }
$$

(Here, we are using that for large enough $N, H^{j}\left(X, \mathcal{O}_{X}(N(d-j)+i)\right.$ vanishes for $0<j<d$ and $H^{j-1}\left(\mathcal{O}_{X}(N(d-j)+i)\right.$ vanishes for $1<j<d$.)

Corollary 3.10. Let $S$ be a Cohen-Macaulay section ring, of dimension $d \geq 2$ and a-invariant a. Let $I=S_{\geq N}$ for $N \gg 0$. Then

$$
S_{\geq N d+a+1} \subset \text { gradedcore }(I) \subset \bigoplus_{i \in \mathbb{Z} ; S_{i}=0} S_{N d+a-i} .
$$

Furthermore, the second inclusion is an equality if $S$ is balanced in the sense that the entire socle of $H_{\mathfrak{m}}^{d}(S)$ lives in degree $a$.

Proof. To prove gradedcore $(I) \subset \bigoplus_{i \in \mathbb{Z} ; S_{i}=0} S_{N d+a-i}$, take any $z \in$ gradedcore $(I)$. Without loss of generality, we may assume that $z$ is homogeneous, say of degree $b$. Take any $w \in \omega_{S}$ of degree precisely $-a$, the minimal possible. Then $z w \in$ gradedcore $\left(I \omega_{S}\right)$ is an element of degree $b-a$. Thus by Theorem 3.1 for some $i$ such that $S_{i}=0$. This degree restriction on $z$ forces $z \in S_{N d+a-i}$. Thus the one inclusion is proved.

The inclusion $S_{\geq d N+a+1} \subset$ gradedcore $(I)$ follows immediately from the second part of Lemma 3.9 Fix any homogeneous system of parameters $x_{1}, \ldots, x_{d}$ of degree $N$. To see that $S_{N d+i} \subset\left(x_{1}, \ldots, x_{d}\right)$, we need the vanishing of $H^{d-1}\left(X, \mathcal{O}_{X}(i)\right)=$ $\left[H_{\mathfrak{m}}^{d}(S)\right]_{i}$, where $X=\operatorname{Proj} S$. For $i \geq a+1$, this follows simply from the definition of the $a$-invariant as the largest $i$ such that $\left[H_{\mathfrak{m}}^{d}(S)\right]_{i}$ is non-zero. When the socle of $H_{\mathfrak{m}}^{d}(S)$ is concentrated in degree a, Lemma 3.9 also implies that $S_{N d+a-i} \subset$ $\left(x_{1}, \ldots, x_{d}\right) S$ whenever $S_{i}=0$. Indeed, we need to check only that $\left[H_{\mathfrak{m}}^{d}(S)\right]_{a-i}$ is zero. But if $\eta$ is a non-zero element of $\left[H_{\mathfrak{m}}^{d}(S)\right]$, then $\eta$ must have a non-zero multiple in the socle $\left[H_{\mathfrak{m}}^{d}(S)\right]_{a}$. But if $\eta$ has degree $a-i$, this is impossible whenever $S_{i}=0$. 
Combining our Theorem 3.1] with the results of [16], we arrive at the following corollary.

Corollary 3.11. Let $\mathcal{L}$ be an ample invertible sheaf on a rationally singular variety $X$. Then $\mathcal{L}$ has a non-zero global section if and only if

$$
\text { gradedcore }\left(I \omega_{S}\right)=\operatorname{adj}\left(I^{d} \omega_{S}\right)
$$

where $S$ is the section ring of the pair $(X, \mathcal{L}), d$ is its dimension, $I$ is the ideal of $S$ generated by all elements of degrees at least $N$, for $N \gg 0$, and $\operatorname{adj}\left(I^{d} \omega_{S}\right)$ is the adjoint module of $I^{d}$ as defined in [16, Remark 6.0.7].

Thus, using [16], Kawamata's conjecture [18] is equivalent to the following conjecture.

Conjecture 3.12. Let $S$ be the section ring of an ample Cartier divisor $D$ on a rationally singular $\mathbb{Q}$-Gorenstein variety $X$. Assume that the $\mathbb{Q}$-Cartier divisor $D-K_{X}$ is ample. Then

$$
\operatorname{gradedcore}\left(I \omega_{S}\right)=\operatorname{adj}\left(I^{d} \omega_{S}\right)
$$

where $I=S_{\geq N}$, for $N \gg 0$.

An interesting special case of Kawamata's conjecture is when $X$ is Fano and $D=-K_{X}$. Then the conjecture collapses to the following.

Conjecture 3.13. Let $S$ be a section ring with Gorenstein rational singularities. Then

$$
\operatorname{gradedcore}(I)=\operatorname{adj}\left(I^{d}\right)
$$

where $I=S_{\geq N}$ for $N \gg 0$. Here $\operatorname{adj}\left(I^{d}\right)$ denotes the adjoint (or multiplier) ideal (as defined, for example, in [21]; see also [22]).

Finally, for completeness, we also include the following result, valid for graded rings of dimension one. The argument is different.

Theorem 3.14. Let $S$ be a one dimensional $\mathbb{N}$-graded Cohen-Macaulay ring defined over a field containing the rational numbers. Suppose that $S$ has homogeneous nonzero-divisors of degree $N$ for all $N \gg 0$. Let $I=S_{\geq N}$ be the ideal generated by all elements of degree at least $N$, where $N \gg 0$. Then gradedcore $\left(I \omega_{S}\right)=\operatorname{core}\left(I \omega_{S}\right)$ if and only if there exists a non-zero-divisor of degree one in $S$. Furthermore, in this case, $\operatorname{core}(I)=$ gradedcore $(I)$.

Proof. We know by [16, Corollary 5.2.1 and Lemmas 3.1.4 and 3.4.5] that for any homogeneous ideal $I$, core $\left(I \omega_{S}\right)=J^{r+1} \omega_{S}: \omega_{S} I^{r}$ for $r \gg 0$, where $J$ is any reduction of $I$, and $r \gg 0$.

In particular, if $J$ is an ideal of $S$ generated by a non-zero-divisor $x$ of degree $N$, then

$$
\operatorname{core}\left(I \omega_{S}\right)=J^{r+1} \omega_{S}: \omega_{S} I^{r} \quad \text { and } \quad \text { gradedcore }\left(I \omega_{S}\right)=J^{r+1} \omega_{S}: \omega_{S} I^{\prime r},
$$

where $I=S_{\geq N}$ is the ideal generated by all elements of degree at least $N$ and $I^{\prime}=S_{N} S$ is the ideal generated by the elements of degree precisely $N$.

Now observe that, as submodules of $\omega_{S}$ tensored with the full ring of quotients of $S$,

$$
J^{r+1} \omega_{S}: \omega_{S} I^{r}=x^{r+1} \operatorname{Hom}_{S}\left(I^{r}, \omega_{S}\right)
$$


and

$$
J^{r+1} \omega_{S}: \omega_{S} I^{\prime r}=x^{r+1} \operatorname{Hom}_{S}\left(I^{\prime r}, \omega_{S}\right) .
$$

So gradedcore $\left(I \omega_{S}\right)=\operatorname{core}\left(I \omega_{S}\right)$ if and only if $\operatorname{Hom}_{S}\left(I^{r}, \omega_{S}\right)=\operatorname{Hom}_{S}\left(I^{\prime r}, \omega_{S}\right)$. But as $S$ is Cohen-Macaulay, this is equivalent to $I^{r}=I^{\prime r}$.

Suppose now that gradedcore $\left(I \omega_{S}\right)=\operatorname{core}\left(I \omega_{S}\right)$. Then $I^{r}=I^{\prime r}$, and so $I^{r}$ is generated in degrees precisely $N r$. In particular, $S_{r N+1}=S_{1} S_{r N}$. This means that $S_{r N+1}$ is contained in the ideal generated by $S_{1}$. Now, as there are non-zero-divisors of degree $r N+1$, there must be a non-zero-divisor of degree one. Otherwise, $S_{N r+1}$ would be contained in a minimal prime and would consist only of zero-divisors.

Conversely, if $S$ admits a non-zero-divisor $y$ of degree one, then there exists a $q$ such that $S_{\geq n}=y^{n-q} S_{\geq q}$ for all $n \geq q$. In particular, for each $p \geq 0$ and $N \geq q$,

$$
\left[I^{r}\right]_{N r+p} \subset S_{N r+p} \subset y^{N r+p-q} S_{q} \subset\left(\left(y^{N}\right)^{r-1} y^{N-q} S_{q}\right) S \subset\left(I^{\prime}\right)^{r} .
$$

Since $I^{r}=\bigoplus_{p \geq 0}\left[I^{r}\right]_{N r+p}$, we conclude that $I^{r}=I^{\prime r}$, and so the core and graded core modules are equal.

For the final statement, assume that $\operatorname{core}\left(I \omega_{S}\right)=$ gradedcore $\left(I \omega_{S}\right)$, and let $z \in$ gradedcore $(I)$. Then $z \omega_{S} \in \operatorname{gradedcore}\left(I \omega_{S}\right)=\operatorname{core}\left(I \omega_{S}\right)$, so that for every reduction $J$ of $I$, we have $z \in J \omega_{S}: S \omega_{S}$. As any such $J$ is generated by a nonzero-divisor, it follows that $z \in J$ for all reductions $J$ of $I$, and so $z \in \operatorname{core}(I)$.

\section{The CORE OF POWERS OF $\mathfrak{m}$ IN A STANDARD GRADED RING}

We now turn to the proof of Theorem 1.3 from the introduction, giving a formula for the core in a reduced standard graded ring of arbitrary characteristic.

Theorem 4.1. Let $(S, \mathfrak{m})$ be a reduced Cohen-Macaulay graded ring generated in degree one over an infinite field. Let d denote the dimension of $S$ and let a denote its a-invariant. Then

$$
\operatorname{core}\left(\mathfrak{m}^{n}\right)=\text { gradedcore }\left(\mathfrak{m}^{n}\right)=\mathfrak{m}^{n d+a+1}
$$

for all $n \in \mathbb{N}$.

Note that there is no restriction on the characteristic of the ground field in Theorem 4.1. This is especially interesting in light of examples from [24], which show in general that the core depends on the characteristic.

Proof. By [2, Theorem 4.5], we know that $\operatorname{core}\left(\mathfrak{m}^{n}\right)=\operatorname{gradedcore}\left(\mathfrak{m}^{n}\right)$, so we consider only the graded core. Lemma 3.9 shows that $\mathfrak{m}^{n d+a+1} \subset$ gradedcore $\left(\mathfrak{m}^{n}\right)$, for all $n$ (not just large $n$ ). (Note that because $S$ has depth at least two, $S_{n}=$ $H^{0}\left(X, \mathcal{O}_{X}(n)\right)$ for all $n$.) It remains to prove the other inclusion.

The point is to prove the analogous statement inside the canonical module of $S$, namely that

$$
\operatorname{gradedcore}\left(\mathfrak{m}^{n} \omega_{S}\right) \subset\left[\omega_{S}\right]_{\geq n d+1} .
$$

This will imply that gradedcore $\left(\mathfrak{m}^{n}\right) \subset S_{\geq n d+a+1}$, as follows. Suppose that $z \in$ gradedcore $\left(\mathfrak{m}^{n}\right)$ has degree less than $n d+a+1$. Choose any $w \in \omega_{S}$ of degree $-a$. Then $z w \in$ gradedcore $\left(\mathfrak{m}^{n} \omega_{S}\right)$ is an element of degree less than $n d+1$, contradicting (5).

To see that gradedcore $\left(\mathfrak{m}^{n} \omega_{S}\right) \subset\left[\omega_{S}\right]_{\geq n d+1}$, we use Lemma 4.2 below, applied to the ideal $I=\mathfrak{m}$. The hypothesis is satisfied by easy degree reasons: since $\Omega_{p}=\left[\omega_{S}\right]_{\geq p+1}$, and $J$ is generated by elements of degree $n$, it follows immediately 
that $\Omega_{n d-1} \cap J \omega_{S}=J \Omega_{n d-1-n}$. Note that the associated graded ring $G$ is simply $S$ again. So to show that

$$
\operatorname{graded} \operatorname{core}\left(\mathfrak{m}^{n}\right) \subset \Omega_{n d}=\left[\omega_{S}\right]_{\geq n d+1}
$$

it suffices to show the vanishing of

$$
\left[\bigcap\left(x_{1}^{*}, \ldots, x_{d}^{*}\right) \omega_{G}\right]_{n d}=\left[\bigcap\left(x_{1}, \ldots, x_{d}\right) \omega_{S}\right]_{n d},
$$

where the intersection is taken over all homogeneous systems of parameters of degree $n$. Now letting $T=S^{(n)}$ denote the $n$-th Veronese subring of $S$, we have

$$
\left[\bigcap\left(x_{1}, \ldots, x_{d}\right) \omega_{S}\right]_{n d}=\left[\bigcap\left(x_{1}, \ldots, x_{d}\right) \omega_{T}\right]_{d} .
$$

Thus we need to prove the vanishing of $\bigcap J \omega_{T}$ in degree $d$ as $J$ ranges over all homogeneous systems of parameters of $T$ of degree one. But since $T$ is a reduced Cohen-Macaulay ring of dimension $d$ generated in degree one, the vanishing of this intersection is an immediate consequence of Lemma 4.3 below.

Lemma 4.2. Let $I \subset A$ be an $\mathfrak{m}$-primary ideal in a local or graded ring $A$ of dimension $d$ at least two, and let $G$ denote the associated graded ring of $A$ with respect to $I$. Fix any $n \in \mathbb{N}$, and let $\mathcal{R}$ be any non-empty set of reductions of $I^{n}$. Assume that $\Omega_{n d-1} \cap J \omega_{A}=J \Omega_{n d-1-n}$ for all $J \in \mathcal{R}$. Then

$$
\bigcap_{\left\{x_{1}, \ldots, x_{d}\right\} \in \mathcal{R}}\left(x_{1}, \ldots, x_{d}\right) \omega_{A} \subset \Omega_{d n}=\Gamma\left(\operatorname{Proj} A[I t], I^{n d} \omega_{\operatorname{Proj} A[I t]}\right)
$$

if

$$
\left[\bigcap_{\left\{x_{1}, \ldots, x_{d}\right\} \in \mathcal{R}}\left(x_{1}^{*}, \ldots, x_{d}^{*}\right) \omega_{G}\right]_{n d}=0,
$$

where $x_{i}^{*}$ denotes the image of $x_{i}$ in $I^{n} / I^{n+1}$, considered as an element of $G$. The converse holds if $G$ is Cohen-Macaulay.

Proof. This is a slight variant of the Key Lemma, [16, Lemma 3.3.1]. The proof is essentially the same, so we omit it.

Lemma 4.3. Let $T$ be a reduced Cohen-Macaulay graded ring of positive dimension d finitely generated by its degree one elements over an infinite field $k$. Then $\bigcap\left(x_{1}, \ldots, x_{d}\right) \omega_{T}$ vanishes in degree $d$, where the intersection is taken over all homogeneous systems of parameters of degree one.

Proof. By induction on $d$ (see [16, proof of Theorem 3.4.1]), we reduce immediately to the case where $T$ has dimension one. Note that in doing this induction, we can kill $d-1$ generic one-forms, so that the resulting one-dimensional ring can be assumed to be reduced. The scheme Proj $T$ is a finite set of reduced points in some projective space $\mathbb{P}^{n}=\mathbb{P}\left(T_{1}^{*}\right)$. Say there are $e$ points.

Now, by Lemma 4.4 below, it suffices to prove the dual statement, namely that the module $H_{\mathfrak{m}_{T}}^{1}(T)$ is generated in degree -1 as a $k$-vector space by elements of the form $\left[\frac{1}{x}\right]$, where $x$ ranges through non-zero-divisors in $T$ of degree one.

Now let $y$ be any non-zero-divisor of degree $e$. Then, thinking of $H_{\mathfrak{m}_{T}}^{1}(T)$ as the direct limit $T / y \longrightarrow T / y^{2} \longrightarrow \ldots$ whose maps are given by multiplication by $y$, it 
is easy to check that

$$
\left[H_{\mathfrak{m}_{T}}^{1}(T)\right]_{-1}=\left[\frac{T_{e-1}}{y}\right] .
$$

We now claim that we can find non-zero-divisors $x_{1}, \ldots, x_{e}$ of degree one such that

$$
\left[\frac{1}{x_{1}}\right], \ldots,\left[\frac{1}{x_{e}}\right]
$$

generate $\left[H_{\mathfrak{m}_{T}}^{1}(T)\right]_{-1}$ as a $k$-vector space. This is equivalent to asking that the elements

$$
\left[\frac{x_{1} \ldots \hat{x}_{i} \ldots x_{e}}{x_{1} \ldots x_{e}}\right] \quad \text { for } i=1, \ldots, e
$$

generate $\left[H_{\mathfrak{m}_{T}}^{1}(T)\right]_{-1}$, which is in turn equivalent to asking that the forms

$$
\ell_{i}=x_{1} \ldots \hat{x}_{i} \ldots x_{e} \quad \text { for } i=1, \ldots, e
$$

span $T_{e-1}$. (Here, the notation $\hat{x}_{i}$ means that $x_{i}$ has been omitted.)

To find such $x_{i}$, just let $x_{i}$ be any linear form on $\mathbb{P}^{n}$ vanishing at the point $P_{i}$ but not at any other point of $\operatorname{Proj} T$. Then $\ell_{i}$ vanishes at all the points except $P_{i}$, and so the $\ell_{i}$ are linearly independent elements of $T_{e-1}$. On the other hand, since the dimension of $T_{e-1}$ is $e$, these elements $\ell_{i} \operatorname{span} T_{e-1}$. The proof is complete.

Lemma 4.4. Let $G$ be any $\mathbb{N}$-graded ring generated in degree one as an algebra over an Artinian local ring $G_{0}$ and satisfying Serre's $S_{2}$ condition. Let d denote the dimension of $G$. Let $\mathcal{R}$ be any set of ideals generated by homogeneous systems of parameters for $G$ of degree one. Then

$$
\left[\bigcap_{J \in \mathcal{R}} J \omega_{G}\right]_{d}=0
$$

if $\left[H_{\mathfrak{m}_{G}}^{d}(G)\right]_{-d}$ is generated over $G_{0}$ by elements of the form

$$
\left[\frac{1}{x_{1} \ldots x_{d}}\right] \text { for }\left(x_{1}, \ldots, x_{d}\right) \in \mathcal{R} \text {. }
$$

The converse holds if $G$ is Cohen-Macaulay.

Proof. We use the natural duality pairing

$$
\left[H_{\mathfrak{M}_{G}}^{d}(G)\right]_{-d} \otimes_{A}\left[\omega_{G}\right]_{d} \longrightarrow\left[E_{G}(k)\right]_{0}=E_{G_{0}}(k)=\omega_{G_{0}},
$$

where $k$ is the residue field of $G$ (and $G_{0}$ ), sending

$$
\eta \otimes \omega \mapsto \omega(\eta) \text {. }
$$

More explicitly, making the identification, $E_{G}(k)=H_{\mathfrak{M}}^{d}\left(\omega_{G}\right)$, we have

$$
\left[\frac{g}{\left(x_{1} \cdots x_{d}\right)^{t}}\right] \otimes \omega \mapsto\left[\frac{g \omega}{\left(x_{1} \cdots x_{d}\right)^{t}}\right] \text {. }
$$

Now, let us first suppose that $\left[H_{\mathfrak{M}_{G}}^{d}(G)\right]_{-d}$ is generated as a $G_{0}$-module by the special elements $\left[\frac{1}{x_{1} \cdots x_{d}}\right]$. Take any $w \in \bigcap\left(x_{1}, \ldots, x_{d}\right) \omega_{G}$ of degree $d$. Then $\langle\eta, w\rangle=0$ for any element $\eta$ of the form

$$
\left[\frac{1}{x_{1} \cdots x_{d}}\right] \text {. }
$$

So, since these generate $\left[H_{\mathfrak{M}_{G}}^{d}(G)\right]_{-d}$, we get $\langle\eta, w\rangle=0$ for all $\eta \in\left[H_{\mathfrak{M}_{G}}^{d}(G)\right]_{-d}$. Since the pairing is perfect, this implies that in fact $w$ is zero, as needed. 
Conversely, suppose that $\bigcap\left(x_{1}, \ldots, x_{d}\right) \omega_{G}$ contains no non-trivial elements of degree $d$. Let $H$ be the $G_{0}$-submodule of $H_{\mathfrak{M}_{G}}^{d}(G)$ generated by the special elements $\left[\frac{1}{x_{1} \cdots x_{d}}\right]$. Consider the exact sequence

$$
0 \longrightarrow H \longrightarrow\left[H_{\mathfrak{M}}^{d}(G)\right]_{-d} \longrightarrow C \longrightarrow 0 .
$$

Taking the Matlis dual over the zero-dimensional ring $G_{0}$, we obtain

$$
\operatorname{Hom}_{G_{0}}\left(C, \omega_{G_{0}}\right)=\operatorname{Ker}\left(\left[\omega_{G}\right]_{d} \longrightarrow \operatorname{Hom}_{G_{0}}\left(H, \omega_{G_{0}}\right)\right) \text {. }
$$

We now compute the kernel of the map $\left[\omega_{G}\right]_{d} \longrightarrow \operatorname{Hom}_{G_{0}}\left(H, \omega_{G_{0}}\right)$. An element $w$ is sent to the zero map $H \longrightarrow \omega_{G_{0}}$ if and only if for all $\eta \in H$, the pairing $\langle\eta, w\rangle=0$. But this occurs if and only if for all $\left(x_{1}, \ldots, x_{d}\right) \in \mathcal{R}$, the element $\left[\frac{w}{x_{1} \cdots x_{d}}\right]$ is zero. Since $\omega_{G}$ is Cohen-Macaulay, this in turn means that $w \in\left(x_{1}, \ldots, x_{d}\right) \omega_{G}$ for all $\left(x_{1}, \ldots, x_{d}\right)$ in $\mathcal{R}$. The proof is complete.

\section{Questions}

In this final section, we gather together a few commutative algebra questions whose positive solutions would solve Kawamata's conjecture, at least in special cases. To keep things simple, we will focus on the case where $H^{i}\left(X, \mathcal{O}_{X}\right)$ vanishes for $i>0$, which simplifies statements considerably. This has the effect of allowing us to assume that the section rings $S$ have rational singularities (see [16, §6]). All of these questions are interesting if we furthermore assume that $S$ is Gorenstein with isolated singularity and $a$-invariant -1 ; this corresponds to the important special case where $\mathcal{L}$ is the anti-canonical divisor on a smooth Fano variety.

Question 1. Suppose that $S$ is a section ring with rational singularities, and let $Y^{\prime} \longrightarrow$ Spec $S$ be the proper birational map obtained by blowing up the ideal generated by all elements of degree $N, N \gg 0$. Let $\nu: Y \longrightarrow Y^{\prime}$ be the normalization map. Then is $\nu_{*} \omega_{Y}=\omega_{Y^{\prime}}$ ? Note that the rational singularities hypothesis implies that at least there is an equality for their global sections.

An affirmative answer to Question 1 is equivalent to the existence of a non-zero element in $S$ of degree one. Indeed, we know that the core module of $I$ is

$$
\Omega_{d}=\Gamma\left(Y, I^{d} \omega_{Y}\right)=\Gamma\left(Y,\left(I^{\prime}\right)^{d} \mathcal{O}_{Y} \otimes \omega_{Y}\right)=\Gamma\left(Y^{\prime},\left(I^{\prime}\right)^{d} \mathcal{O}_{Y^{\prime}} \otimes \nu_{*} \omega_{Y}\right),
$$

so if $\nu_{*} \omega_{Y}=\omega_{Y^{\prime}}$, then this is also equal to

$$
\Gamma\left(Y^{\prime},\left(I^{\prime}\right)^{d} \otimes \omega_{Y^{\prime}}\right)=\Omega_{d}^{\prime},
$$

which is the graded core module of $I$.

The map $Y \longrightarrow Y^{\prime}$ is an isomorphism except along a single irreducible divisor $E^{\prime}$ isomorphic to $\operatorname{Proj} S$. Furthermore, because $\nu_{*} \omega_{Y}$ is $S_{2}$, it suffices to check the isomorphism $\nu_{*} \omega_{Y} \longrightarrow \omega_{Y^{\prime}}$ generically along $E^{\prime}$. Note also that the preimage of $E^{\prime}$ in $Y$ is a single irreducible divisor $E$, also isomorphic to $\operatorname{Proj} S$.

Question 1 can be rephrased more algebraically as follows:

Question 2. Let $S$ be a section ring with rational singularities. Let $I^{\prime}$ be the ideal generated by all elements of degree $N$, for $N \gg 0$, and consider the normalization map $S\left[I^{\prime} t\right] \subset S[I t]$. Then is the naturally induced trace map of graded canonical modules

$$
\omega_{S[I t]} \subset \omega_{S\left[I^{\prime} t\right]}
$$


an isomorphism? Equivalently, is it an isomorphism in sufficiently high degrees, or after localizing at the height one prime containing $I^{\prime} S\left[I^{\prime} t\right]$ ?

Note that when $S_{1} \neq 0$, the map (17) is an isomorphism by Theorem 3.5. Thus in this setting, settling Question 1 or 2 is essentially equivalent to Kawamata's conjecture.

It is worth pointing out that in Questions 1 and 2, we cannot assume, nor can we hope to show in general, that $Y^{\prime}$ satisfies Serre's $S_{2}$ condition:

Proposition 5.1. Let $S$ be a section ring of a normal variety with respect to an ample invertible sheaf $\mathcal{L}$, and assume that $S_{1} \neq 0$. Let $I^{\prime}$ be the ideal generated by elements of degrees precisely $N$, for $N \gg 0$. Then if Proj $S\left[I^{\prime}\right]$ satisfies Serre's $S_{2}$-condition, then $S_{1}$ generates an $\mathfrak{m}$-primary ideal. In particular, $\mathcal{L}$ is globally generated.

Proof. By Theorem 3.5 and Lemma 3.7 we see that if $S_{1} \neq 0$, the inclusion $\omega_{S^{\natural}} \subset$ $\omega_{S^{+}}$is an isomorphism in positive degrees. This forces $\nu_{*} \omega_{Y} \cong \omega_{Y^{\prime}}$. In particular, we have an isomorphism $\left(\nu_{*} \omega_{Y}\right)_{E^{\prime}} \cong\left(\omega_{Y^{\prime}}\right)_{E^{\prime}}$ along the reduced exceptional divisor $E^{\prime}$. This means that the induced trace map for the map of one-dimensional local rings $\mathcal{O}_{Y^{\prime}, E^{\prime}} \hookrightarrow \mathcal{O}_{Y, E}$ is an isomorphism. Let $Q$ be the cokernel of the injection $\mathcal{O}_{Y^{\prime}, E^{\prime}} \hookrightarrow \mathcal{O}_{Y, E}$; note that $Q$ has dimension zero. We have an induced exact sequence of local cohomology modules

$$
0 \longrightarrow Q=H_{\mathfrak{m}}^{0}(Q) \longrightarrow H_{\mathfrak{m}}^{1}\left(\mathcal{O}_{Y^{\prime}, E^{\prime}}\right) \longrightarrow H_{\mathfrak{m}}^{1}\left(\mathcal{O}_{Y, E}\right) \longrightarrow 0,
$$

where $\mathfrak{m}$ is the maximal ideal of $\mathcal{O}_{Y^{\prime}, E^{\prime}}$. Dualizing, we see that the dual of $Q=$ $H_{\mathfrak{m}}^{0}(Q)$ is the cokernel of the trace map. Hence if the trace map in an isomorphism, then $Q$ is zero and the map $\mathcal{O}_{Y^{\prime} E^{\prime}} \hookrightarrow \mathcal{O}_{Y, E}$ is an isomorphism. This shows that $Y^{\prime}$ is regular along $E^{\prime}$, and hence regular everywhere in codimension one.

Now if $Y^{\prime}$ is also $S_{2}$, then it must be normal, whence $Y=Y^{\prime}$. But then, since $Y=\operatorname{Proj} S[I t]$ and $Y^{\prime}=\operatorname{Proj} S\left[I^{\prime} t\right]$, it follows that $I^{r}=I^{\prime r}$ for $r$ sufficiently large. Thus $S_{\geq N r}=S_{N r} S$ for large $r$. In particular, $S_{N r+1}=S_{N r} S_{1}$, which means that the ideal generated by $S_{N r+1}$ is contained in the ideal generated by $S_{1}$. Since $S_{N r+1}$ generates an $\mathfrak{m}$-primary ideal, it follows that so does $S_{1}$. The proof is complete.

This proof shows that the isomorphism $\nu_{*} \omega_{Y} \cong \omega_{Y^{\prime}}$ holds if and only if the induced map of one-dimensional local rings $\mathcal{O}_{Y^{\prime}, E^{\prime}} \hookrightarrow \mathcal{O}_{Y, E}$ is an isomorphism. Since this is the normalization map, we see that $\mathcal{L}$ has a section if and only if the scheme $Y^{\prime}$ is regular in codimension one. Thus we can ask:

Question 3. If $S$ is a rationally singular section ring and $I^{\prime}$ is the ideal generated by all elements of degrees precisely $N$, for $N \gg 0$, then is $\operatorname{Proj} S\left[I^{\prime} t\right]$ regular in codimension one? Equivalently, is the local ring $S\left[I^{\prime} t\right]_{P}$ regular, where $P$ is the unique height one prime containing $I^{\prime} S\left[I^{\prime} t\right]$ ?

Another possible approach to Question 1 is the following.

Question 4. Let $S$ be a rationally singular section ring of dimension $d \geq 2$. Fix $N \gg 0$. Let $I^{\prime}$ be the ideal generated by elements of degree precisely $N$, and let $L$ be the ideal generated by all elements of degrees at least $N d$. Consider the multi-Rees ring $R=S\left[I^{\prime} t\right][L u]$, and let $\mathfrak{M}$ denote its unique homogeneous maximal ideal. Then does $H_{\mathfrak{M}}^{d+1}(R)$ vanish? Equivalently, does it vanish in u-degree zero? 
Interestingly, the trace map for the normalization map of $R$ is an isomorphism. So $\omega_{R}=\omega_{S[I t][L u]}$, and in particular, $R$ has a Cohen-Macaulay canonical module.

An affirmative solution to Question 4 is equivalent to an affirmative answer to Question 1. To see this, first note that for $N \gg 0$, we can assume the cohomology module $H_{\mathfrak{M}}^{d+1}(R)$ is concentrated in $u$-degree zero. Now the point is essentially that $Y$ is obtained from $Y^{\prime}$ by blowing up the sheaf of ideals $L \mathcal{O}_{Y^{\prime}}$. One specific way to see the equivalence is to note that the $u$-degree zero part of $H_{\mathfrak{M}}^{d+1}(R)$ is precisely the dual of the cokernel of the trace map $\Gamma\left(\omega_{\operatorname{Proj} R}\right) \longrightarrow \omega_{\operatorname{Spec} S\left[I^{\prime} t\right]}$, where the scheme Proj $R$ is defined via the $u$-grading on $R$. But now it is easy to check that $\operatorname{Proj} R \cong \mathcal{S p e c}_{Y} \operatorname{Sym}\left(\mathcal{O}_{\operatorname{Proj} S[L t]}(1)\right)$, so that there is a smooth map Proj $R \longrightarrow Y$ realizing Proj $R$ as a line bundle over the smooth variety $Y=\operatorname{Proj} S[L t]$. Thus, one computes directly (as in [16, Proposition 6.2.1]) that $\Gamma\left(\omega_{\operatorname{Proj} R}\right)$ is $\bigoplus_{p \geq 1} \Gamma\left(Y, I^{p} \omega_{Y}\right)$. So the trace $\Gamma\left(\omega_{\operatorname{Proj} R}\right) \longrightarrow \omega_{\text {Spec } S\left[I^{\prime} t\right]}$ is

$$
\bigoplus_{p \geq 1} \Gamma\left(Y, I^{p} \omega_{Y}\right)=\bigoplus_{p \geq 1} \Omega_{p} \hookrightarrow \bigoplus_{p \geq 1} \Gamma\left(Y^{\prime}, I^{\prime p} \omega_{Y^{\prime}}\right)=\bigoplus_{p \geq 1} \Omega_{p}^{\prime} .
$$

But as we know, the trace map $\nu: Y \longrightarrow Y^{\prime}$ is an isomorphism if and only if we have equalities $\Omega_{p}^{\prime}=\Omega_{p}$ for all $p \geq 1$.

We now consider a slightly different direction.

Question 5. Let $S$ be a rationally singular section ring of dimension $d \geq 2$. Set $S^{\dagger}=\bigoplus_{p \in \mathbb{N}} S_{p} S$ as in Section 2. Is the a-invariant of $S^{\dagger}$ negative? That is, does $\omega_{S^{\dagger}}$ vanish in non-positive degrees?

The interest in Question 3 stems from the following.

Proposition 5.2. Let $S$ be an equidimensional section ring of dimension $d \geq 2$ with an isolated non-Cohen-Macaulay point. Then $S_{1} \neq 0$ if and only if $a\left(S^{\dagger}\right)<0$.

Proof. Consider $\omega_{S^{\dagger}}$ as a bigraded module; its homogeneous components are given by Theorem 3.5. In particular, if $S_{1}=0$, then for $p>0$, the degree $(p,-1)$ component of $\omega_{S^{\dagger}}$ is $\left[\omega_{S}\right]_{p-1}$, and so in particular is non-zero. By $p-q$ symmetry, then also the degree $(-1, p)$ component of $\omega_{S^{\dagger}}$ is non-zero. Thus $\omega_{S^{\dagger}}$ does not vanish in $p$-degree -1 , and so $a\left(S^{\dagger}\right) \geq 1$.

Conversely, if $a\left(S^{\dagger}\right)<0$, then $\omega_{S^{\dagger}}$ vanishes in $p$ degree -1 . In particular, $\left[\omega_{S^{\dagger}}\right]_{-1, q}$ is zero, and by symmetry then $\left[\omega_{S^{\dagger}}\right]_{q,-1}$ is zero as well. From Theorem [3.5, it follows that $S_{1}$ must be non-zero. The proof is complete.

Finally, we mention one more group of questions, stemming from our approach to computing the core in [16]. First a bit a notation: For any $\mathfrak{m}$-primary ideal in a local (or graded) ring $(A, \mathfrak{m})$ of dimension $d>0$, let $G=A / I \oplus I / I^{2} \oplus \ldots$ be the associated graded ring of $I$. For any element $x \in I \backslash I^{2}$, let $x^{*}$ denote the class of $x$ in $I / I^{2}$, considered as a degree one element of $G$. Elements of the local cohomology module $H_{\mathfrak{M}_{G}}^{d}(G)$ can be denoted in the usual way as generalized fractions

$$
\left[\frac{g}{\left(x_{1}^{*} \cdots x_{d}^{*}\right)^{t}}\right]
$$

where $g \in G$ and $t \in \mathbb{N}$, and $x_{1}, \ldots, x_{d}$ is a minimal reduction of $I$. Note that the degree of such an element is $\operatorname{deg} g-d t$.

Question 6. Let $S$ be a rationally singular section ring and let $I$ be the ideal generated by all elements of degrees at least $N$, for $N \gg 0$. Let $G=S / I \oplus I / I^{2} \oplus$.. 
be the associated graded ring of $S$ with respect to $I$. Then is $\left[H_{\mathfrak{M}_{G}}^{d}(G)\right]_{-d}$ generated as an $G_{0}$-module by elements of the form

$$
\left[\frac{1}{x_{1}^{*} \cdots x_{d}^{*}}\right]
$$

where $x_{1}, \ldots, x_{d}$ ranges through all homogeneous systems of parameters of degree $N$ ?

Lemma 5.3 below motivates this question. Indeed, this lemma implies that if Question 6 has an affirmative answer, then

$$
\text { gradedcore }\left(I \omega_{S}\right)=\Omega_{d}=\left[\omega_{S}\right]_{\geq N d+1},
$$

so by Theorem 3.1, $S_{1}$ must be non-zero. Note that in the setting of Question 6, the hypothesis of Lemma 5.3 holds by [16, Proposition 4.14]. Interestingly, the main results of 16 imply that Question 6] does have an affirmative solution if we allow $x_{1}, \ldots, x_{d}$ to range over all reductions of $I$, not just homogeneous ones.

Lemma 5.3. Let $I$ be an $\mathfrak{m}$-primary ideal in a local (or graded) ring $(A, \mathfrak{m})$ of dimension $d \geq 2$, and let $G$ be the corresponding associated graded ring $A / I \oplus$ $I / I^{2} \oplus \ldots$ Assume that $G$ satisfies Serre's $S_{2}$ condition. Let $\mathcal{R}$ be any set of minimal reductions of $I$ with $\Omega_{d-1} \cap J \omega_{A}=J \Omega_{d-2}$ for all $J \in \mathcal{R}$. Then

$$
\bigcap_{J \in \mathcal{R}} J \omega_{A} \subset \Omega_{d}
$$

if $\left[H_{\mathfrak{M}_{G}}^{d}(G)\right]_{-d}$ is generated as an $A / I$-module by elements of the form

$$
\left[\frac{1}{x_{1}^{*} \cdots x_{d}^{*}}\right]
$$

where $\left(x_{1}, \ldots, x_{d}\right)$ ranges through all the reductions in $\mathcal{R}$. The converse holds if $G$ is Cohen-Macaulay.

Proof. Using Lemma 4.4, this is essentially a dual form of the Key Lemma of [16] Lemma 3.3.1], which states that under the same hypothesis,

$$
\bigcap_{J \in \mathcal{R}} J \omega_{A}=\Omega_{d} \quad \text { if } \quad \bigcap_{\left(x_{1}, \ldots, x_{d}\right) \in \mathcal{R}}\left[\left(x_{1}^{*}, \ldots, x_{d}^{*}\right) \omega_{G}\right]_{d}=0,
$$

and conversely if $G$ is Cohen-Macaulay.

One approach to Question [6 would be to find an affirmative answer to the next question.

Question 7. Let $S$ be a rationally singular section ring of dimension $d \geq 2$, and let $I$ be the ideal generated by elements of degree exactly $N$, for $N \gg 0$. Denote by

$$
F=S / \mathfrak{m} \oplus I / \mathfrak{m} I \oplus I^{2} / \mathfrak{m} I^{2} \oplus \ldots
$$

the fiber ring of I (whose associated projective scheme defines the scheme-theoretic closed fiber of the map Proj $S[I t] \longrightarrow$ Spec $S$ ), and let $F_{\text {red }}$ denote $F$ modulo its nilradical. Is the map of local cohomology

$$
H_{\mathfrak{m}_{F}}^{d}(F) \longrightarrow H_{\mathfrak{m}_{F}}^{d}\left(F_{\text {red }}\right)
$$

a bijection in degree $-d$ ? 
To see the relationship with Question [6] first note that $H_{\mathfrak{M}_{F}}^{d}(F) \cong H_{\mathfrak{M}_{G}}^{d}(G) \otimes_{S}$ $S / \mathfrak{m}$, where $\mathfrak{m}$ is the maximal ideal of $S$. Thus by Nakayama's lemma, the required elements generate $\left[H_{\mathfrak{M}_{G}}^{d}(G)\right]_{-d}$ as an $S / I$-module if and only if their images modulo $\mathfrak{m}$ generate $\left[H_{\mathfrak{M}_{F}}^{d}(F)\right]_{-d}$ over $k$. So in Question 6 , we might as well ask the analogous question for $F$. On the other hand, it is easy to check that $F_{\text {red }}$ (and $G_{\text {red }}$ ) is isomorphic to $S^{(N)}$, the $N$-th Veronese subring of the section ring $S$. Now because $F_{\text {red }}$ is a reduced standard graded Cohen-Macaulay algebra, Lemma 4.3 ensures that an affirmative answer to Question 7 will imply one for Question [6.

\section{REFERENCES}

[1] F. Ambro, Ladders on Fano varieties, Algebraic Geometry, 9, J. Math. Sci. (New York) 94 (1999), no. 1, 1126-1135. MR 2000e:14067

[2] A. Corso, C. Polini, and B. Ulrich, The structure of the core of ideals, Math. Ann. 321 (2001), no. 1, 89-105. MR 2002j:13005

[3] - Core and residual intersections of ideals, Trans. Amer. Math. Soc. 354 (2002), 2579-2594. MR 2003b:13035

[4] S. D. Cutkosky, Appendix to [22], Math. Res. Lett. 1 (1994), no. 6, 739-755. MR 95k:13028

[5] M. Demazure, Anneaux gradués normaux, Introduction à la théorie des singularités, II, 35-68, Travaux en Cours, 37, Hermann, Paris, 1988. MR 91k:14004

[6] S. Goto and K. Nishida, The Cohen-Macaulay and Gorenstein Rees algebras associated to filtrations, Mem. Amer. Math. Soc. 526 (1994). MR 95b:13001

[7] S. Goto and K. I. Watanabe, On graded rings I, J. Math. Soc. Japan 30 (1978), no. 2, 179-213. MR 81m:13021

[8] S. Goto and Yamagishi, The theory of unconditioned strong d-sequences and modules of finite local cohomology, preprint

[9] A. Grothendieck and J. Dieudonné, Eléments de géométrie algébrique II, Inst. Hautes Etudes Sci. Publ. Math. 8 (1961). MR 29:1208, MR 36:177b

[10] P. Schenzel, N. V. Trung, and N. T. Cuong, Verallgemeinerte Cohen-Macaulay Moduln, Math. Nachr. 85 (1978), 57-73. MR 80i:13008

[11] C. Huneke and I. Swanson, Cores of ideals in 2-dimensional regular local rings, Michigan Math. J. 42 (1995), 193-208. MR 96j:13021

[12] C. Huneke and K. Smith, Tight closure and the Kodaira vanishing theorem, J. Reine Angew. Math. 484 (1997), 127-152. MR 98e:13007

[13] C. Huneke and N. V. Trung, On the core of ideals, preprint.

[14] E. Hyry, Blow-up algebras and rational singularities, Manuscripta Math. 98 (1999), 377-390. MR 2001d:13002

[15] Coefficient ideals and the Cohen-Macaulay property of Rees algebras, Proc. Amer. Math. Soc. 129 (2001), 1299-1308. MR 2001h:13005

[16] E. Hyry and K. E. Smith, On a non-vanishing conjecture of Kawamata and on the core of an ideal, preprint (2002).

[17] S. Itoh, Integral closures of ideals generated by regular sequences, J. Algebra 117 (1988), 390-401. MR 90g:13013

[18] Y. Kawamata, On effective non-vanishing and base-point-freeness, Asian J. Math 4 (2000), 173-181. MR 2002b:14010

[19] Semipositivity, vanishing and applications, School on Vanishing Theorems and Effective Results in Algebraic Geometry, Abdus Salam International Centre for Theoretical Physics, Trieste (2000). (unpublished)

[20] S. Kleiman, Towards a Numerical Theory of Ampleness, Annals of Mathematics (2) 84 (1966), 293-344. MR 34:5834

[21] R. Lazarsfeld, Positivity in Algebraic Geometry, in preparation.

[22] J. Lipman, Adjoints of ideals in regular local rings, Math. Res. Letters 1 (1994), 739-755. MR 95k:13028

[23] Cohen-Macaulayness in graded algebras, Math. Res. Letters 1 (1994), 149-157. MR 95d:13006

[24] C. Polini, and B. Ulrich, A formula for the core of an ideal, preprint. 
[25] D. Rees and J. Sally, General elements and joint reductions, Michigan Math. J. 35 (1988), 241-254. MR 89h:13034

[26] J. B. Sancho de Salas, Blowing-up morphisms with Cohen-Macaulay associated graded rings, Géométrie algébrique et applications, I (La Rábida, 1984), 201-209, Travaux en Cours, 22, Hermann, Paris, 1987. MR 88k:14008

[27] P. Schenzel, Dualisierende Komplexe in der lokalen Algebra und Buchsbaum-Ringe, Lecture Notes in Mathematics, vol. 907, Springer-Verlag, Berlin-New York, 1982. MR 83i:13013

[28] E. Snapper, Multiples of divisors, Jour. of Math. and Mech. 8 (1959), 967-992. MR 22:44

[29] K. E. Smith, Fujita's freeness conjecture in terms of local cohomology, J. Algebraic Geom. 6 (1997), no. 3, 417-429. MR 98m:14002

[30] _ Globally F-regular varieties: Applications to vanishing theorems for quotients of Fano varieties, Michigan Math J. 48 (2000), 553-572. MR 2001k:13007

[31] N. V. Trung, Toward a theory of generalized Cohen-Macaulay modules, Nagoya Math. J. 102 (1986), 1-49. MR 87h:13018

[32] _ The largest non-vanishing degree of graded local cohomology modules, J. Algebra 215 (1999), no. 2, 481-499. MR 2000f:13038

[33] W. V. Vasconcelos, Arithmetic of blowup algebras, London Math. Soc. Lecture Note Ser., vol. 195, Cambridge University Press, Cambridge, 1994. MR 95g:13005

Department of Mathematics, University of Helsinki, Helsinki, Finland

E-mail address: Eero.Hyry@helsinki.fi

Department of Mathematics, University of Michigan, Ann Arbor, Michigan 481091109

E-mail address: kesmith@umich.edu 